\title{
Diferencias en los accidentes laborales en España según país de procedencia del trabajador
}

\author{
Eduardo Rubiales-Gutiérrez, MPH, ${ }^{(1)}$ Andrés A Agudelo-Suárez, MPH, (2),(3) \\ María José López-Jacob, MPH, ${ }^{(4)}$ Elena Ronda-Pérez, PhD. ${ }^{(2)}$
}

Rubiales-Gutiérrez E,Agudelo-Suárez AA, López-Jacob MJ, Ronda-Pérez E.

Diferencias en los accidentes laborales en España según país de procedencia del trabajador. Salud Publica Mex 20 10;52:199-206.

\begin{abstract}
Resumen
Objetivo. Comparar los accidentes laborales en trabajadores inmigrantes y autóctonos en España. Material y métodos. La fuente de información procede de la Encuesta Española de Condiciones de Trabajo. La nacionalidad del trabajador fue la variable explicativa principal, clasificada según el índice de desarrollo humano (IDH). La variable resultado fueron los accidentes laborales $(\mathrm{AL})$. Otras variables incluidas fueron sociodemográficas y condiciones de empleo. Se calcularon prevalencias y razones de momios (RM) crudas y ajustadas (regresión logística) con sus intervalos de confianza al 95\% (IC95\%). Resultados. La prevalencia de AL en trabajadores de países de bajo IDH fue $12.7 \%$ (mujeres: II.I\%), y en españoles $10.3 \%$ (mujeres: $8.1 \%$ ). Comparado con trabajadoras autóctonas, las mujeres de países de bajo IDH presentaron mayor riesgo (RM ajustada I.66; IC95\% I.2I-2.28). Conclusión. Es necesario profundizar en el análisis causal de los $\mathrm{AL}$ en inmigrantes identificando los posibles factores de riesgo y reforzando las medidas preventivas para su control.
\end{abstract}

Palabras clave: inmigrantes; condiciones de trabajo; accidentes de trabajo; encuestas; España
Rubiales-Gutiérrez E,Agudelo-Suárez AA, López-Jacob MJ, Ronda-Pérez E.

Differences in occupational accidents in Spain according to the worker's country of origin. Salud Publica Mex 2010;52:199-206.

\begin{abstract}
Objective. Compare the occupational accidents between autochthonous and immigrant workers in Spain. Material and Methods. Data were obtained from the Spanish Survey of Working Conditions. Nationality was considered as an explicative variable, and the country of origin was regrouped according to the Human Development Index-HDI. Occupational accidents were the outcome. Other variables included were sociodemographic and employment conditions. Prevalences were calculated as well as simple and adjusted odds ratio (OR) (logistic regression) with a $95 \%$ confidence interval (Cl 95\%). Results. The prevalence of occupational accidents was $12.7 \%$ (women, II.I\%) for workers from low HDI countries and $10.3 \%$ (women, 8.1\%) for Spaniards. A higher risk of occupational accidents was observed among women from low HDI countries compared to Spaniards (adjusted OR I.66;95\%Cl I.2I-2.28). Discussion. It is necessary to conduct a more thorough causal analysis of occupational accidents among immigrants in order to identify risk factors and strengthen prevention and control strategies.
\end{abstract}

Key words: immigrants; working conditions; occupational accidents; surveys; Spain

(I) Universidad Miguel Hernández. Elche, España.

(2) Área de Medicina Preventiva y Salud Pública. Universidad de Alicante, España.

(3) Facultad de Odontología. Universidad de Antioquia. Medellín, Colombia.

(4) Instituto Sindical de Trabajo,Ambiente y Salud. España.

Fecha de recibido: 30 de julio de 2009 - Fecha de aceptado: 13 de enero de 2010 Solicitud de sobretiros: Andrés A Agudelo-Suárez. Área de Medicina Preventiva y Salud Pública. Universidad de Alicante Campus de San Vicente de Raspeig s/n. 03690 Alicante. España.

Correo electrónico: agudeloandres@odontología.udea.edu.co; oleduga@gmail.com 
$\mathrm{L}^{2}$ tendencia ascendente en los últimos años de población inmigrante en España, especialmente procedente de países con una baja renta per cápita, y motivada fundamentalmente por la búsqueda de mejoras socioeconómicas y de calidad de vida o huyendo de la inseguridad política de sus países, ${ }^{1,2}$ supone un nuevo reto para la administración pública encargada de evaluar y planificar las políticas en seguridad y salud laboral. Así, teniendo en cuenta los datos de afiliación a la seguridad social del Ministerio de Trabajo e Inmigración a junio de 2009, ${ }^{3} 1929937$ de los trabajadores son extranjeros (65\% provienen de países diferentes de la Unión Europea); lo que equivale a $10 \%$ de toda la población trabajadora afiliada en el país.

Sin embargo, la evidencia científica sobre el impacto que las condiciones de trabajo y de empleo pueden tener sobre la salud de los inmigrantes en España es hasta ahora insuficiente. ${ }^{4}$ Algunas publicaciones en salud laboral e inmigración han puesto de manifiesto las diferencias existentes entre las condiciones de trabajo y empleo del trabajador nacido en España y del trabajador inmigrante, señalando una mayor precariedad laboral, ${ }^{5,6}$ que influye en la percepción de riesgos laborales ${ }^{6,7}$ y del estado de salud de los trabajadores extranjeros. ${ }^{8}$ En el caso de los accidentes laborales, una revisión de la literatura científica realizada a partir de 48 artículos encontró mayores tasas de lesión por accidente de trabajo en extranjeros con respecto a trabajadores autóctonos; ${ }^{9}$ además, estas diferencias se intensificaban en determinadas ocupaciones y en trabajadores recién llegados al país.

En España se han realizado algunas investigaciones utilizando la información de accidentes de trabajo procedentes de las estadísticas oficiales, ${ }^{10-12}$ las que han evidenciado mayor riesgo de accidentes laborales en trabajadores extranjeros. En estos estudios se comprueba que las diferencias se mantienen al analizar por sector económico separadamente. Además, por ejemplo, en el caso del servicio doméstico se tiene un régimen de seguridad social especial que no incluye la contingencia de accidentes de trabajo; y existen muchos otros casos de economía sumergida donde la mayor parte de las trabajadoras no están dadas de alta, por lo tanto no se cotiza y no se declaran los accidentes. ${ }^{7}$ Las explicaciones argumentadas por los autores se basan en la adjudicación de puestos más peligrosos dentro de cada sector, mayor exposición (tiempo de trabajo), peor conocimiento (información, formación) y mayor vulnerabilidad, que les limitaría la posibilidad de protestar o rechazar trabajos peligrosos. No obstante, estos estudios sólo dan información sobre aquellos accidentes en trabajadores afiliados a la seguridad social que tienen cubierta de forma específica esta contingencia, y dejan fuera de su alcance a algunos trabajadores autónomos y trabajado- res informales de la economía sumergida, sectores que en España emplean un gran número de inmigrantes. ${ }^{4}$ Por otra parte, las dificultades para obtener datos desagregados por nacionalidad en estas estadísticas limitan la profundidad de los análisis en relación con aquellos trabajadores que son considerados inmigrantes.

Las encuestas de condiciones de trabajo y salud se han convertido en una herramienta esencial en la identificación de las condiciones de trabajo y cómo afectan éstas en la salud de la población trabajadora tanto a nivel nacional como internacional. ${ }^{13,14}$ En el caso del estado español es el Instituto de Seguridad e Higiene en el Trabajo, a instancias del Ministerio del Trabajo e Inmigración, el encargado de desarrollar periódicamente esta tarea a través de la Encuesta Nacional de Condiciones de Trabajo (ENCT). ${ }^{14,15}$ Entre otras variables recoge información sobre los accidentes que ocurren en el lugar de trabajo al margen de que se haya o no notificado oficialmente, y no solamente los que tienen la consideración legal de accidente de trabajo; ${ }^{16}$ además, el conjunto de la encuesta permite una visión global de las condiciones de trabajo. La incorporación de información sobre el país de nacionalidad del trabajador desde 2008 permite realizar análisis más detallados, como el que se plantea en este estudio, cuyo objetivo es comparar los accidentes laborales entre la población inmigrante y autóctona en España.

\section{Material y métodos}

\section{Diseño y fuentes de información}

Se trata de un estudio transversal. La información procede de la VI Encuesta Nacional de Condiciones de trabajo (ENCT 2008). Los microdatos fueron facilitados por el Instituto Nacional de Seguridad e Higiene en el Trabajo y se utilizó el cuestionario general del trabajador (disponible en la página electrónica del Instituto Nacional de Seguridad e Higiene en el Trabajo). ${ }^{16,17}$ También en esta dirección se puede consultar el diseño técnico de la encuesta. La población del estudio estuvo compuesta por los trabajadores ocupados de todas las actividades económicas, pertenecientes a todo el territorio nacional, a excepción de Ceuta y Melilla, con un total de 18518444 trabajadores. La información se obtuvo por entrevista en el domicilio del trabajador escogido. El trabajo de campo fue realizado entre el 12 de diciembre de 2006 y el 11 de abril de 2007.

La muestra fue de 11054 trabajadores (6 471 hombres y 4583 mujeres). Sin embargo, para efectos de comparación, el análisis se restringió a la población con información completa sobre el país de nacionalidad con bajo índice de desarrollo humano, por lo que la 
muestra final incluida fue de 10927 trabajadores (6 401 hombres), $98.9 \%$ del total.

\section{Variables}

La nacionalidad del trabajador fue la variable principal de exposición. Se obtuvo de la pregunta P.71 de la ENCT 2008. Las categorías de esta pregunta son: 1) Española y 2) Extranjera (especificando el país). La nacionalidad de los trabajadores extranjeros fue reclasificada en función del índice de desarrollo humano (IDH) de los diferentes países; y se consideraron como países con bajo $\operatorname{IDH}(<0.9)$, aquellos países de Europa, Latinoamérica, Asia y Oceanía que no entraron en la categoría de IDH muy alto del Programa de las Naciones Unidas para el Desarrollo (PNUD). ${ }^{18}$

Se consideró como variable de resultado los accidentes laborales (AL). Dicha información fue obtenida de la pregunta P.59: “En los dos últimos años, ¿ha sufrido algún accidente en su trabajo que requiera asistencia médica o tratamiento, o la aplicación de primeros auxilios? (Sí/No).

Otras variables incluidas en el estudio fueron los riesgos de accidente percibidos que existen durante el desarrollo del trabajo (respuesta múltiple con 20 ítems), edad ( 16 a 24, 25 a 44, 45 a 64, 65 y más), sexo, nivel de estudios (sin estudios/primarios, secundarios/bachiller o universitarios), situación de trabajo (autónomo, asalariado con alta/sin alta), tipo de contrato (fijo/ temporal,) tiempo de contrato (parcial/ completo), sector de actividad (público/privado), ocupación principal (profesionales, directivos, administrativos, comerciantes, servicios, agricultura, manuales de industria y construcción), horas trabajadas a la semana, jornada de trabajo (fija/partida, o turnos), características del horario de trabajo (trabaja sábados, domingos / festivos y si el horario se adapta a compromisos sociales y familiares), aspectos del trabajo o empleo (opinión sobre el salario, posibilidades de ascenso, relaciones personales, oportunidades de aprender y prosperar).

\section{Análisis de los datos}

En primer lugar se realizó un análisis descriptivo de frecuencias absolutas y relativas de las variables del estudio. Estas variables fueron ponderadas utilizando los coeficientes de ponderación establecidos para cada ciudad en la base de datos. Asimismo se obtuvieron prevalencias de riesgos de accidentes por nacionalidad, sexo y cálculo de $R M$ según variables sociodemográficas. Se realizaron pruebas $\mathrm{ji}^{2}$ para diferencia de proporciones en riesgos laborales y prevalencias de accidentes laborales. Por último, se estimó la asociación de la nacionalidad con $\mathrm{AL}$, por medio de cálculo de razones de momios simples y ajustadas con sus respectivos intervalos de confianza al 95\% (IC95\%) por regresión logística (SPSS 15.0). Se tomó como grupo de referencia a los de nacionalidad española. Se incluyeron como variables de control: sexo, edad, nivel educativo, situación de trabajo actual, tipo de contrato, sector y ocupación.

La información y los métodos empleados en este trabajo corresponden a un estudio de riesgo mínimo y cumplen con las especificaciones éticas para la investigación en seres humanos. El protocolo que dio origen al manuscrito fue evaluado por el Área de Medicina Preventiva y Salud Pública de la Universidad de Alicante (España).

\section{Resultados}

El cuadro I muestra las características sociodemográficas de los trabajadores incluidos en el estudio. La mayor proporción de mujeres trabajadoras proviene de países con bajo IDH, así como la población trabajadora menor de 44 años. La proporción de personas con estudios superiores es mayor en trabajadores españoles; en el caso de los extranjeros de países de bajo IDH la mayoría tienen estudios secundarios (42.1\%).

El cuadro II presenta las condiciones de empleo en los dos grupos. En líneas generales, la mayoría de los

Características sociodemográficas de la población DE TRABAJADORES INCLUIDOS EN EL ESTUDIO SOBRE ACCIDENTES LABORALES SEGÚN PAíS DE PROCEDENCIA ( $\mathrm{N}=1$ 0927). EsPAÑA, 2008

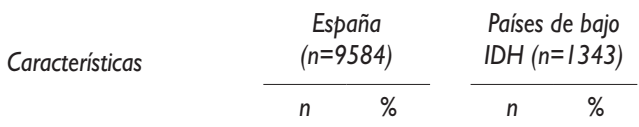

Sexo

\begin{tabular}{lllll} 
Hombre & 5625 & 58.7 & 774 & 57.7 \\
\hline Mujer & 3959 & 41.3 & 569 & 42.3
\end{tabular}

Edad

\begin{tabular}{lrrrr}
16 a 24 & 900 & 9.4 & 183 & 13.6 \\
\hline 25 a 44 & 5632 & 58.8 & 943 & 70.2 \\
\hline 45 a 64 & 2990 & 31.2 & 211 & 15.8 \\
\hline 65 y más & 40 & 0.4 & 3 & 0.2 \\
\hline No contesta & 22 & 0.2 & 3 & 0.2
\end{tabular}

Nivel educativo

$\begin{array}{llllll}\text { Sin estudios/estudios primarios } & 3776 & 39.4 & 453 & 33.7\end{array}$

$\begin{array}{lllll}\text { Estudios secundarios/bachiller } & 3582 & 37.4 & 566 & 42.1\end{array}$

\begin{tabular}{lrrrr} 
Estudios universitarios & 2139 & 22.3 & 275 & 20.4 \\
\hline
\end{tabular}

$\begin{array}{lrrrr}\text { No contesta } & 88 & 0.9 & 49 & 3.7\end{array}$




\section{Cuadro II \\ Condiciones de EMPLeO de los tRabajadores SEGÚN PAíS DE PROCEDENCIA ( $\mathrm{N}=\mid$ | 0927). ESPAÑA, 2008}

Características

n $\%$

Situación de trabajo actual

Asalariado con alta en la seguridad social

\begin{tabular}{rrrr}
1785 & 18.6 & 124 & 9.2 \\
\hline 7484 & 78.1 & 1031 & 76.7 \\
\hline 275 & 2.9 & 179 & 13.3 \\
\hline 37 & 0.4 & 9 & 0.6 \\
4 & 0.0 & 1 & 0.0
\end{tabular}

Otro

No sabe/no contesta

$\begin{array}{rr}5546 & 74 \\ 1864 & 24 \\ 66 & 0\end{array}$

$\begin{array}{rrr}74.1 & 565 & 54.9 \\ 24.9 & 456 & 44.4 \\ 0.9 & 7 & 0.7 \\ 0.1 & 0 & 0.0\end{array}$

No sabe/no contesta

1067

14.27

147

14.3

Parcial

6413

85.73

88

85.7

Tipo de sector

Privado

8092

1262

Otro

No sabe/no contesta

177
53

84.4

13.2

53

1.8

127|

\begin{tabular}{lr}
71 & 94.7 \\
\hline 43 & 3.2 \\
\hline 17 & 1.3 \\
\hline 12 & 0.9
\end{tabular}

Ocupación principal

Profesionales

1600

Directivas

Administrativas

Comerciantes

Servicios

Agricultura

Manuales de industria y construcción

No sabe/No contesta

\begin{tabular}{rrrr}
1600 & 16.7 & 58 & 4.3 \\
539 & 5.6 & 26 & 1.9 \\
1099 & 11.5 & 49 & 3.7 \\
1358 & 14.2 & 138 & 10.2 \\
\hline 2367 & 24.7 & 576 & 42.9 \\
\hline 454 & 4.7 & 83 & 6.2 \\
\hline 2155 & 22.5 & 411 & 30.6 \\
\hline 12 & 0.1 & 2 & 0.2
\end{tabular}

Número de horas trabajadas/semana

\begin{tabular}{|c|c|c|c|c|}
\hline$<=20$ & 702 & 7.3 & 92 & 6.9 \\
\hline $2 \mathrm{I}-40 \mathrm{~h}$ & 6276 & 65.5 & 767 & 57.1 \\
\hline$<=41$ & 2530 & 26.4 & 451 & 33.6 \\
\hline No sabe/no contesta & 76 & 0.8 & 33 & 2.5 \\
\hline
\end{tabular}

Jornada de trabajo

Partida (mañana y tarde)

Fija (mañana, tarde o noche)

4138

Turnos

Otro

No sabe/no contesta

$3039 \quad 31.7$

$2169 \quad 22.6$

$202 \quad 2.1$

2.1

553

446

281

54

Horario de trabajo (porcentajes no excluyentes)

Trabajo sábados (Sí)

\begin{tabular}{rrrr}
5148 & 53.7 & 867 & 64.5 \\
\hline 2903 & 30.3 & 490 & 36.5 \\
\hline 7302 & 76.2 & 953 & 71
\end{tabular}

Se adapta a compromisos sociales y familiares (Sí)

7302

76.2

953

Aspectos del trabajo/empleo (porcentajes no excluyentes)

Opinión sobre el salario (Bueno)

Buenas posibilidades de ascenso profesional (Si)

Relaciones personales positivas (Sí)

Oportunidades de aprender y prosperar (Sí)

4052

3351

7297

5340

42.3

35

55.7

\begin{tabular}{ll}
532 & 39.6 \\
\hline 397 & 29.6 \\
\hline 970 & 72.2 \\
\hline 649 & 48.3
\end{tabular}

* (n=7028 para España y 1028 para países de bajo IDH) 
trabajadores están asalariados con alta en la Seguridad Social, si bien existe un porcentaje de trabajadores de países de bajo IDH que no lo están (13.3\%). Atendiendo al tipo de contrato, la temporalidad en los trabajadores de bajo IDH es superior a la de los españoles (44.4\%), y la duración de la jornada es de más de $40 \mathrm{~h} /$ semana $(33.6 \%)$. De igual manera son los trabajadores de países de bajo IDH los que mayoritariamente se emplean en las actividades de la construcción (30.6\%) y los servicios $(42.9 \%)$.

En relación con los riesgos percibidos de accidentes laborales en población trabajadora (cuadro III), los hombres de países de bajo IDH refieren mayor riesgo de cortes y pinchazos $(39.1 \%)$, golpes $(25.5 \%)$ y caídas de personas desde cierta altura (28.4\%). Las mujeres de países de bajo IDH refieren mayor riesgo de caídas de personas al mismo nivel $(23.4 \%$ ) y quemaduras $(12.5 \%)$. En estos riesgos existen diferencias estadísticamente significativas en las proporciones con respecto a los trabajadores españoles $(p<0.05)$. Por otro lado, son los trabajadores autóctonos los que indican tener más riesgo de sufrir accidentes de tráfico (hombres: $18 \%$ y mujeres: $5.3 \%$ ) y atracos, agresiones físicas $u$ otros actos violentos (mujeres: 7.3\%). Estas diferencias también son estadísticamente significativas con respecto a los trabajadores de bajo IDH $(p<0.05)$.

El cuadro IV muestra las prevalencias de accidentes laborales y las RM (IC95\%), según diversas características sociodemográficas y nacionalidad. Son los trabajadores de países de bajo IDH (12.7\%), las mujeres (11.1\%) y los de edad entre 45 y 64 años $(17.5 \%$ ) de estos países los que presentaron las prevalencias más altas con diferencias significativas con respecto a los trabajadores españoles $(p<0,05)$. Asimismo, los trabajadores de estos países con estudios universitarios (13.5\%), con contratos temporales $(15.4 \%)$, con alta en la seguridad social (14.7\%) y contratados por el sector privado $(12.9 \%)$ refirieron mayor prevalencia de accidentes laborales $(p<0.05)$.

Por último, con respecto a los resultados del análisis simple y multivariado (figura 1), después de ajustar por las diferentes variables de control, la población de países de bajo IDH presentó mayor riesgo de lesiones

\section{Cuadro III}

\section{Prevalencia de Riesgos de accidentes laborales Percibidos en trabajadores SEGÚN PAÍS DE PROCEDENCIA ( $\mathrm{N}=\mid$ 0927). EsPAÑA, 2008}

\begin{tabular}{|c|c|c|c|c|}
\hline \multirow[b]{2}{*}{ Riesgos } & \multicolumn{2}{|c|}{ Hombres $(n=6399)$} & \multicolumn{2}{|c|}{ Mujeres $(n=4528)$} \\
\hline & $\begin{array}{l}\text { Españoles } \\
(n=5625)\end{array}$ & $\begin{array}{l}\text { Países bajo IDH } \\
\quad(n=774)\end{array}$ & $\begin{array}{l}\text { Españolas } \\
(n=3959)\end{array}$ & $\begin{array}{l}\text { Países bajo IDH } \\
\quad(n=569)\end{array}$ \\
\hline Caídas de personas desde cierta altura & 19.9 & $28.4^{*}$ & 6.9 & $9.5^{*}$ \\
\hline Caídas de personas & 18.7 & $24.3^{*}$ & 18.7 & $23.4^{*}$ \\
\hline Caídas de objetos, materiales o herramientas & 17.0 & $25.5^{*}$ & 6.7 & 7.6 \\
\hline Desplomes o derrumbamientos & 7.7 & $14.7^{*}$ & I.I & I.I \\
\hline Cortes y pinchazos & 30.8 & $39.1 *$ & 25.2 & 25.7 \\
\hline Golpes & 31.2 & $37.0 *$ & 19.3 & 20.0 \\
\hline Atropellos, atrapamientos o aplastamientos por vehículos & $9.5^{*}$ & 6.1 & 1.9 & 0.7 \\
\hline Atrapamientos o aplastamientos con equipos o maquinaria & 9.3 & 10.7 & 1.5 & 2.5 \\
\hline Proyección de partículas o trozos de material & 8.1 & $10.9 *$ & 1.0 & 0.5 \\
\hline Quemaduras (contacto con superficies calientes, con productos químicos, etc.) & 10.0 & 9.8 & 9.4 & $12.5^{*}$ \\
\hline $\begin{array}{l}\text { Daños producidos por un exceso de exposición al sol (quemaduras, insolación, golpe } \\
\text { de calor) }\end{array}$ & 4.3 & $5.9 *$ & 1.0 & $0.0^{\ddagger}$ \\
\hline Incendios & 3.6 & 3.7 & 1.9 & 2.3 \\
\hline Explosiones & 2.6 & 1.8 & 0.6 & I.I \\
\hline $\begin{array}{l}\text { Daños producidos por animales (mordeduras, coces, picotazos, picaduras de insectos, } \\
\text { etc.) }\end{array}$ & 2.1 & 1.4 & I.I & 0.5 \\
\hline $\begin{array}{l}\text { Contactos eléctricos (líneas de alta tensión, conexiones, cables o enchufes en mal } \\
\text { estado...) }\end{array}$ & 6.6 & 6.1 & 3.1 & 3.0 \\
\hline Sobreesfuerzos por manipulación manual de cargas & 10.1 & $12.9 *$ & 7.0 & 7.4 \\
\hline Intoxicación por manipulación de productos tóxicos & 5.0 & 4.0 & $3.9 *$ & 2.1 \\
\hline Accidentes de tráfico & $18.0^{*}$ & 9.7 & $5.3^{*}$ & 2.1 \\
\hline Atracos, agresiones físicas u otros actos violentos & 7.4 & 7.2 & $7.2^{*}$ & 3.7 \\
\hline
\end{tabular}




\section{Cuadro IV}

Prevalencia de accidentes laborales y RM (IC95\%) POR CARACTERísticas SOCIOdemográficas Y DE EMPLEO SEGÚN EL PAís DE PROCEDENCIA DEL TRABAJADOR ( $\mathrm{N}=10927)$. EsPAÑA, 2008

\section{Características sociodemográficas}

\begin{tabular}{|c|c|c|c|}
\hline \multicolumn{4}{|c|}{ Accidentes laborales } \\
\hline España $(n=9584)$ & $\begin{array}{c}\text { Países de bajo IDH } \\
\quad(n=1343)\end{array}$ & $R M$ & IC95\% \\
\hline$\%$ & $\%$ & & \\
\hline
\end{tabular}

Total

10.3

$12.7^{*}$

1.28

I.07- 1.52

Sexo

\begin{tabular}{|c|c|c|c|c|}
\hline Hombre & 11.8 & 14.0 & 1.22 & $0.98-|.5|$ \\
\hline Mujer & 8.1 & II.I* & I.4I & $1.06-1.88$ \\
\hline
\end{tabular}

Edad

\begin{tabular}{|c|c|c|c|c|}
\hline 16- 24 & 10.6 & 9.8 & 0.92 & $0.54-1.57$ \\
\hline 25- 44 & 10.1 & 12.1 & 1.23 & $0.99-1.53$ \\
\hline 45- 64 & 10.6 & $17.5^{*}$ & 1.82 & I.25- 2.64 \\
\hline$<65$ & 12.5 & $0.0^{\ddagger}$ & 2.10 & $0.18-23.2$ \\
\hline
\end{tabular}

Nivel educativo

\begin{tabular}{|c|c|c|c|c|}
\hline Sin estudios/estudios primarios & 12.5 & 11.9 & 0.95 & $0.71-1.29$ \\
\hline Estudios secundarios/bachiller & 10.0 & 11.5 & 1.17 & $0.88-1.55$ \\
\hline Estudios universitarios & 6.7 & $13.5^{*}$ & 2.18 & I.48- 3.20 \\
\hline
\end{tabular}

Tipo de contrato*

\begin{tabular}{|c|c|c|c|c|}
\hline Fijo-permanente & 11.9 & 1.3 & 1.25 & $0.98-1.61$ \\
\hline Temporal & 9.7 & $15.4^{*}$ & 1.64 & $1.22-2.20$ \\
\hline Otro & 15.2 & 12.5 & 0.81 & $0.09-7.35$ \\
\hline
\end{tabular}

Situación de trabajo actual

\begin{tabular}{|c|c|c|c|c|}
\hline Asalariado con alta en la seguridad social & $1 \mathrm{I} .4$ & $14.7 *$ & 1.35 & $1.12-1.63$ \\
\hline Asalariado sin alta en la seguridad social & 6.2 & $1.7^{*}$ & 0.26 & $0.07-0.90$ \\
\hline Autónomo & 6.7 & $12.1 *$ & 1.98 & $|| 2-3.5 \mid$. \\
\hline
\end{tabular}

Tipo de sector

\begin{tabular}{|c|c|c|c|c|}
\hline Público & 12.4 & 9.3 & 0.74 & $0.26-2.11$ \\
\hline Privado & 10.0 & $12.9 *$ & 1.33 & I.I2- 1.60 \\
\hline Otro & 7.9 & 11.8 & 1.55 & $0.32-7.49$ \\
\hline
\end{tabular}

Ocupación principal

\begin{tabular}{|c|c|c|c|c|}
\hline Profesionales & 7.3 & 10.3 & 1.49 & $0.62-3.54$ \\
\hline Directivas & 5.4 & 7.7 & 1.53 & $0.34-6.80$ \\
\hline Administrativas & 5.1 & 0.0 & 0.38 & $0.05-2.80$ \\
\hline Comerciantes & 6.7 & 5.8 & 0.86 & $0.4|-| .8 \mid$ \\
\hline Servicios & 13.6 & 13.4 & 0.98 & $0.75-1.28$ \\
\hline Agricultura & 9.7 & 12.0 & 1.25 & $0.60-2.60$ \\
\hline Manuales de industria y construcción & 15.0 & 16.5 & 1.12 & $0.84-1.49$ \\
\hline
\end{tabular}

El grupo de referencia son los españoles en cada categoría (I.00)

* Diferencia de proporciones entre España/países con bajo IDH $p<0.05$

‡ No aplica 


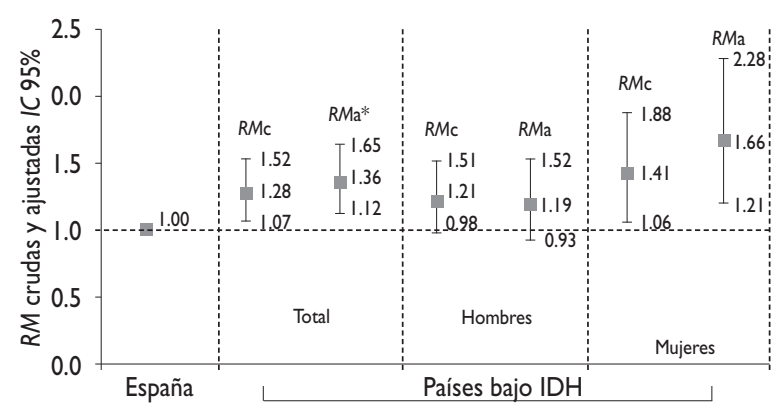

RMc RM crudas

RMa RM ajustadas por edad, nivel educativo, situación de trabajo actual, tipo de contrato, sector y ocupación.

RMa* Ajustada por todas las variables y por sexo.

Figura I. RM CRUDAS Y AJUSTADAS DE ACCIDENTES LABORALES SEGÚN EL PAíS DE PROCEDENCIA DEL TRABAJADOR Y SEXO $(\mathrm{N}=10927)$. EsPaña, 2008

por accidentes laborales (RMa 1.36 IC95\% 1.12- 1.65), y especialmente en las mujeres (RMa 1.66 IC95\% 1.212.28), comparado con la población española.

\section{Discusión}

Los principales hallazgos de esta investigación muestran un mayor riesgo de AL en la población extranjera que procede de países de bajo IDH con respecto a la autóctona. En estos trabajadores, la prevalencia más elevada se encontró en mujeres, en trabajadores jóvenes, en aquellos con contratos temporales, en el sector privado y en ocupaciones de menor cualificación. De otra parte, los principales riesgos referidos por los trabajadores de estos países se asociaron a diferentes tipos de caídas y de cortes y golpes, los cuales son más elevados en comparación con la población española.

En términos generales, se encontró un mayor riesgo de sufrir accidentes laborales en la población procedente de países en desarrollo, aun después de ajustar por las variables de control. Este resultado es similar a otros estudios realizados en España. ${ }^{10-12}$ El mayor riesgo de accidentes en el trabajo en la población inmigrante en España podría ser explicado por el hecho de que en muchas ocasiones son estos trabajadores los que ocupan puestos de menor cualificación, ${ }^{6}$ en condiciones menos ventajosas, ${ }^{5}$ de precariedad ${ }^{4}$ y mayor inseguridad laboral. ${ }^{19,20}$ De hecho, fueron los trabajadores más jóvenes, con menor experiencia laboral, y con contratación temporal entre el colectivo de trabajadores inmigrantes los que tuvieron mayor prevalencia de accidentes laborales, mostrando diferencias que fueron significativas con los trabajadores españoles.

La utilización de encuestas para la observación de la ocurrencia y tendencias de los accidentes de trabajo enriqueció el conocimiento de estos eventos al constituir estrategias complementarias y al incorporar información que no está presente en los partes oficiales. Asimismo, permitió desarrollar análisis diferentes y profundizar en aspectos que caracterizan mejor a los colectivos más afectados. ${ }^{21}$ Además, la metodología de la encuesta realizada en hogares permitió conocer las lesiones de personas de las que no se registran los accidentes, ya sea por que no se puede al no estar en los regímenes de la seguridad social que facultan para ello, como es el caso de la mayoría de los autónomos o del servicio doméstico (con una elevada presencia de trabajadoras extranjeras), o porque están en situación que limita su declaración-vulnerabilidad, desconocimiento-. El uso de la ENCT permitió observar a estos colectivos más "invisibles" para las estadísticas oficiales.

Es importante tener en cuenta que las mayores prevalencias de accidentes laborales se encontraron en los trabajadores inmigrantes de ocupaciones manuales, los cuales se encuentran en sectores de actividad con altas demandas físicas y mayores jornadas de trabajo (servicios y construcción). Situación explicada por los tipos de riesgos referidos por esta población, los cuales fueron mayormente de carácter físico, y esto se relaciona con el tipo de trabajo que desempeñan. ${ }^{22}$

Las diferencias en las prevalencias de accidentes en mujeres trabajadoras inmigrantes respecto a las españolas son muy destacables y permiten intuir un riesgo diferencial en función de la ocupación que desempeñan. Las mujeres inmigrantes generalmente se ubican en el sector doméstico y la hostelería. ${ }^{6}$ En el primer caso, podrían estar sometidas a diferentes tipos de riesgos físicos y químicos, ${ }^{7}$ y menores medidas de protección que explicarían en parte la mayor exposición a riesgo de lesiones por accidentes de tipo laboral. ${ }^{20}$

Se encontró un porcentaje de población inmigrante sin alta en la seguridad social, que puede reflejar un número importante de población extranjera en situación irregular, como algunos estudios plantean. ${ }^{23}$ La situación legal se constituye, por lo tanto, como un factor asociado a la ocurrencia de los accidentes laborales en inmigrantes, en concordancia con la evidencia disponible. ${ }^{24}$

Entre las limitaciones de los hallazgos se encuentra el sesgo de recuerdo de los encuestados, ya que el periodo temporal hace referencia a dos años y puede ocurrir que los accidentes más leves no se declaren. No obstante, en cualquier caso podría haber una subestimación del efecto. Por otra parte, las ENCT no recogen el país de nacimiento del trabajador, sino la 
nacionalidad, por lo que puede darse el caso de una mala clasificación. Hay que destacar como una de las fortalezas del estudio el carácter aleatorio de la encuesta y la representatividad de toda la geografía nacional, así como la realización de las entrevistas en el propio domicilio, lo que mejora la calidad de respuesta.

En el campo de la salud laboral y salud pública, en el caso español todavía son muchas las preguntas por resolver sobre el tema de inmigración, trabajo y salud, lo cual sugiere nuevas hipótesis y nuevos estudios que se están desarrollando en la actualidad, sobre todo dirigidos a los determinantes de por qué se producen más accidentes. ${ }^{25}$ Los resultados encontrados sugieren la necesidad de profundizar en la accesibilidad de las medidas de información, en el respeto a los derechos laborales, así como de dirigir de forma especial los programas de prevención de accidentes en el trabajo a este colectivo.

\section{Agradecimientos}

Al Instituto Nacional de Seguridad e Higiene en el Trabajo por facilitar los microdatos para la realización de este estudio. Este manuscrito es parte del proceso de formación de AA Agudelo-Suárez en el doctorado en Salud Pública y será usado como parte del material empleado para la disertación de la tesis doctoral en la Universidad de Alicante.

\section{Referencias}

I. Jansà JM. Inmigración y envejecimiento, nuevos retos en salud pública. Gac Sanit 2006;20:10-4.

2. Arango J. La inmigración en España en el siglo XXI. En: Maldonado J. Informe sobre la situación demográfica en España. España: Fundación Fernando Abril Martorell, 2004:161-186.

3. Ministerio de Trabajo e Inmigración. Extranjeros con tarjeta o autorización de residencia. [Documento en Internet] Madrid: Ministerio de trabajo; 2009. [consultado 2009 julio 2I] Disponible en: http:// extranjeros.mtas.es/es/InformacionEstadistica/

4. Porthé V,Amable M, Benach J. La precariedad laboral y la salud de los inmigrantes en España: ¿qué sabemos y qué deberíamos saber? Arch Prev Riesgos Labor 2007; 10: 34-39.

5. Ahonen EQ, Porthé V,Vázquez ML, García AM, López-Jacob MJ, RuizFrutos $C$, et al.A qualitative study about immigrant workers perceptions of their working conditions in Spain.J Epidemiol Community Health 2009;63(II):936-942.

6. García AM, López-Jacob MJ,Agudelo-Suárez AA, Ruíz-Frutos C, Porthé V. Condiciones de trabajo y salud en inmigrantes (proyecto ITSAL): entrevistas a informantes clave. Gac Sanit 2009; 23: 91-99.

7. Ahonen EQ, López-Jacob MJ,Vázquez ML, PorthéV, Gil-González D, García AM, et al. Invisible work, unseen hazards:The health of women immigrant household service workers in Spain. Am J Ind Med 2009; [Epub]. Disponible en: http://www3.interscience.wiley.com/journal//22408537/ abstract.
8. Agudelo-Suárez A, Ronda-Pérez E, Gil-González D,Vives-Cases C. Percepción sobre condiciones de trabajo y salud de la población inmigrante colombiana en Alicante, España. Rev Salud Publica (Bogota) 2008;10:160-167.

9. Ahonen EQ, Benavides FG, Benach J. Immigrant populations, work and health - a systematic literature review. Scand JWork Environ Health 2007; 33:96-104.

10. Ahonen EQ, Benavides FG. Risk of fatal and non-fatal occupational injury in foreign workers in Spain.J Epidemiol Community Health 2006; 60: 424-426.

II. Benavides FG, Ahonen EQ, Bosch C. Riesgo de lesión por accidente laboral en trabajadores extranjeros (España, 2003 y 2004). Gac Sanit 2008; 22:44-47.

12. López-Jacob MJ,Ahonen EQ, García AM, Gil A, Benavides FG. Comparación de las lesiones por accidente de trabajo en trabajadores extranjeros y españoles por actividad económica y comunidad autónoma (España, 2005). Rev Esp Salud Pública 2008; 82: 179-187

I3. Rantanen J, Kauppinen T, Toikkanen J, Kurppa K, Lehtinen S, Leino T.Work and Health country profiles - Country profiles and national surveillance indicator in occupational health and safety. People and work, Research reports, 44. Helsinki: Finnish Institute of Occupational Health, 2001. 14. Narocki C, Zimmermann N,Artazcoz L, Gimeno D, Benavides FG. Encuestas de condiciones de trabajo y salud en España: comparación de los contenidos del cuestionario del trabajador.Arch Prev Riesgos Labor 2009; 12:60-68.

15. Instituto Nacional de Seguridad e Higiene en el trabajo.VI Encuesta Nacional de Condiciones de Trabajo. Madrid: Instituto Nacional de Seguridad e Higiene en el trabajo, 2008.

16. Garrido A, Ruiz-Frutos C. Notificación, investigación y registro de accidentes de trabajo. En: Salud Laboral. Barcelona: Masson; 2007: 234-244. 17. Instituto Nacional de Higiene y Seguridad en el Trabajo [sitio de Internet] Madrid: Instituto Nacional de Higiene y Seguridad en el Trabajo. [Consultado 2009 Junio 2I]. Disponible en; http://www.insht.es/ 18. Programa de las Naciones Unidas para el Desarrollo (PNUD). Estadísticas del Informe sobre Desarrollo Humano [sitio de internet]. Nueva York: Programa de las Naciones Unidas para el Desarrollo [consultado 2009 Mayo 20] Disponible en: http://hdr.undp.org/es/ estadisticas/

19. McKay S, Craw M, Chopra D. Migrant workers in England and Wales. An assessment of migrant worker health and safety risks. [Monografía de Internet] Norwich: Health and Safety Executive, 2006. [Consultado 2009 Junio 20] Disponible en: http://www.hse.gov.uk/research/rrpdf/rr502.pdf 20. Agudelo-Suárez AA, Gil-González D, Ronda-Pérez E, Porthé V, Paramio-Pérez G, García AM, et al. Discrimination, work and health in immigrant populations in Spain. Soc Sci Med 2009; 68: 1866- 1874.

2I. Baker EL, Honchar PA, Fine LJ. Surveillance in occupational illness and injury: concepts and content. Am J Public Health 1989; 79: 9-II.

22. España- Ministerio de trabajo y asuntos sociales. Condiciones laborales de los trabajadores inmigrantes en España 2007. [Monografía de internet] España: Ministerio de trabajo y asuntos sociales [consultado 2009 mayo 29]. Disponible en: http://www.seg-social.es/stpri00/groups/public/ documents/binario//00598.pdf

23. McCauley LA. Immigrant workers in the United States: Recent trends, vulnerable populations, and challenges for occupational health.AAOHN ] 2005; 53:313-319.

24. Izquierdo-Escribano A, Fernández-Suárez B. La inmigración en España 2005-2006: entre la normalización y el flujo de cayucos. En: La Situación Social en España (II).Viçent Navarro (dir). Madrid: Fundación F. Largo Caballero, 2007:219-256.

25. García AM, López-Jacob M, Sousa E, Benavides FG. Accidentes de trabajo en una muestra de trabajadores inmigrantes en España. En: Memorias XIII Congreso SESPAS, Sevilla (España: 4-6 de marzo 2009). Gac Sanit 2009; 23 (Esp I) I: 88. 